\title{
New Insights into the Structure of Exotic Nuclei Using the RISING Active Stopper
}

\author{
P.H.Regan ${ }^{\mathrm{a}}$, N.Alkhomashi ${ }^{\mathrm{a}}$, N.Al-Dahan ${ }^{\mathrm{a}}, Z_{\text {s.Podolyák }}$, E.B.Suckling ${ }^{\mathrm{a}}$, \\ P.D.Stevenson ${ }^{\mathrm{a}}$, S.B.Pietri ${ }^{\mathrm{a}, \mathrm{b}}$, S.J.Steer ${ }^{\mathrm{a}}$, A.B.Garnsworthy ${ }^{\mathrm{a}, \mathrm{d}}$, W.Gelletly ${ }^{\mathrm{a}}$, \\ J.Benlliure ${ }^{\mathrm{c}}$, A.I.Morales ${ }^{\mathrm{c}}$, J.Gerl ${ }^{\mathrm{b}}$, M.Górska ${ }^{\mathrm{b}}$, H.J.Wollersheim ${ }^{\mathrm{b}}$, \\ R.Kumar $^{\mathrm{b}, \mathrm{e}}$, J.Grebosz ${ }^{\mathrm{f}}$, A.Algora ${ }^{\mathrm{g}, \mathrm{h}}$, G.Benzoni ${ }^{\mathrm{i}}$, P.Boutachkov ${ }^{\mathrm{b}}$, \\ A.M.Bruce', E.Casarejos ${ }^{c}$, I.J.Cullen ${ }^{\mathrm{a}}$, A.M.Denis Bacelar ${ }^{\mathrm{j}}$, A. Blazhev ${ }^{\mathrm{k}}$, \\ M.E.Estevez ${ }^{\mathrm{c}, \mathrm{g}}$, G.Farrelly ${ }^{\mathrm{a}}$, Y.Fujita ${ }^{1}$, R.Hoischen ${ }^{\mathrm{m}}$, S.Lalkovski ${ }^{\mathrm{j}}$, Z.Liu $^{\mathrm{n}}$, \\ I.Kojouharov $^{\mathrm{b}}$, N.Kurz ${ }^{\mathrm{b}}$, C.Mihai ${ }^{\circ}$, F.Molina ${ }^{\mathrm{g}}$, D.Mücher ${ }^{\mathrm{k}}$, B.Rubio ${ }^{\mathrm{g}}$, \\ H.Schaffner ${ }^{\mathrm{b}}$, S.Tashenov ${ }^{\mathrm{b}}$, A.Tamii ${ }^{\mathrm{p}}$, J.J. Valiente Dobón ${ }^{\mathrm{q}}$, P.M.Walker ${ }^{\mathrm{a}}$, \\ P.J.Woods ${ }^{\mathrm{m}}$
}

aDepartment of Physics, University of Surrey, Guildford, GU2 7XH,

${ }^{b}$ GSI, Planckstrasse 1, D-64291, Darmstadt, Germany

${ }^{c}$ Universidad de Santiago de Compostela, E-15706, Santiago de Compstela, Spain

${ }^{d}$ TRIUMF, 4004 Wesbrook Mall, Vancowver, BC V6T 2A3, Canada

'Inter University Accelerator Centre, New Delhi, India

${ }^{f}$ The Henryk Niewodniczanski Institute of Nuclear Physics, PL-31-342, Krakow, Poland

${ }^{8}$ Instituto de Fisica Corpuscular, CSIC - Universidad de Valencia, Spain, E-46071, Spain

${ }^{h} M T A$ ATOMKI, Debrecen, Hungary

${ }^{i} I N F N$, Universitá degli Studi di Milano, I-20133, Milano, Italy

${ }^{j}$ School of Environment and Technology, University of Brighton, Brighton BN2 4GJ, UK

${ }^{k} I K P$, Universitat zu Köln, D-50937, Koln, Germany

'Department of Physics, Osaka University, Toyonaka, Osaka, 560-0043, Japan

${ }^{m}$ Department of Physics, Lund University, Lund, S-22100, Lund, Sweden

${ }^{n}$ Department of Physics and Astronomy, University of Edinburgh, Edinburgh, UK

${ }^{\circ}$ National Institute for Physics and Nuclear Engineering, RO-077125, Bucharest, Romania

${ }^{p}$ Research Centre for Nuclear Physics, Osaka University, Ibaraki, Osaka 567-0047, Japan

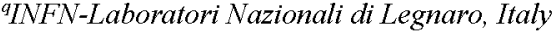

\begin{abstract}
This conference paper outlines the operation and some of the preliminary physics results using the GSI RISING active stopper. Data are presented from an experiment using combined isomer and beta-delayed gamma-ray spectroscopy to study low-lying spectral and decay properties of heavy-neutron-rich nuclei around A 190 produced following the relativistic projectile fragmentation of ${ }^{208} \mathrm{~Pb}$ primary beam. The response of the RISING active stopper detector is demonstrated for both the implantation of heavy secondary fragments and in-situ decay of beta-particles. Beta-delayed gamma-ray spectroscopy following decays of the neutronrich nucleus ${ }^{194} \mathrm{Re}$ is presented to demonstrate the experimental performance of the set-up. The resulting information inferred from excited states in the $\mathrm{W}$ and Os daughter nuclei is compared with results from Skyrme Hartree-Fock predictions of the evolution of nuclear shape.
\end{abstract}

Keywords: Beta-decay, exotic nuclei, projectile fragmentation

PACS: $21.10 .-\mathrm{k} ; 21.60 . \mathrm{JZ} ; 23.40 .-\mathrm{s} ; 27.90 .+\mathrm{b}$

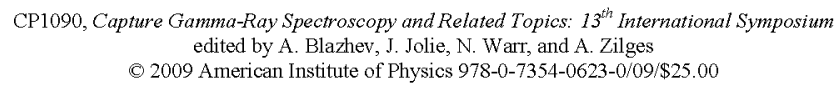




\section{INTRODUCTION}

The use of high-energy beams of radioactive nuclei produced following relativistic projectile fragmentation and/or fission reactions allows the production and study of previously unreachable regions of the Segre chart [1]. Using magnetic separators, such as the FRagment Separator (FRS) at GSI [2], highly exotic nuclei can be distinguished and selected from a background of more intensely produced, less exotic secondary products from such reactions often allowing the very first spectroscopic information on excited states to be obtained for such nuclei. Previous studies which have combined this radioactive beam production technique with high-resolution gamma-ray spectroscopy measurements include measurements of decays from isomeric states in a wide range of both neutron-rich and -deficient nuclei [3-13]. While this technique has proved to be an extremely powerful tool for many nuclei, it is clearly limited to systems which have isomeric states in the suitable range of lifetimes. This paper focuses on the first results using the Stopped RISING Active Stopper which allows correlations between exotic heavy-ions produced by relativistic fragmentation/fission reactions and their subsequent beta-decays, thereby allowing beta-delayed spectroscopy to be performed in the daughter nucleus. The combination of decay spectroscopy using both isomeric and beta-delayed correlations opens up the possibilities for studies of a range of nuclear systems for the first time. The current work concentrates on the most neutron-rich nuclei studied to date in the Ta, $\mathrm{W}$ and $\mathrm{Re}$ isotopes. Other related studies have been made using the same Active Stopper set-up for very neutron-rich nuclei around the $\mathrm{N}=126$ [14] and also to study Gamow-Teller decays along the $\mathrm{N}=\mathrm{Z}$ line [15].

\section{THE STOPPED RISING EXPERIMENTAL CONFIGURATION}

The RISING gamma-ray array [16] is placed at the focus of the GSI fragment separator (see Fig. 1) and consists of fifteen, high efficiency seven-element germanium cluster detectors which can be arranged in different geometries to allow studies of (i) fast fragmentation beams [17] and (ii) decay studies $[18,19]$ using a close, compact set-up. The latter represents the Stopped RISING set-up $[10,11,13,18,19]$. In this configuration, the germanium detectors are placed in three rings of 5 clusters, providing a photo-peak efficiency of $15 \%$ at $662 \mathrm{keV}$. Details of the response of the RISING gamma-ray array in its Stopped Beam configuration can be found in references $[18,19]$. The RISING array surrounds the Active Stopper.

\section{The RISING Active Stopper}

The RISING Active Stopper [20] consists of up to 9 Double-Sided Silicon Strip Detectors (DSSSDs), each with 16 horizontal and vertical strips respectively. Each DSSSD was $5 \mathrm{~cm}$ by $5 \mathrm{~cm}$ in overall dimension and $1 \mathrm{~mm}$ thick and was used to measure the energy and position of the implanted secondary fragments of interest from the projectile fragmentation reactions and their subsequent beta-particles following the 
radioactive decays of the nuclei of interest and their daughters. The ultimate aim of the device is to correlate beta-decays with specific exotic radioactive mother nuclei on an event-by-event basis. This allows both a direct measurement of the lifetime of the betadecay and, using beta-gamma coincident gamma-ray measurements, structural studies in the daughter nuclei to be made.
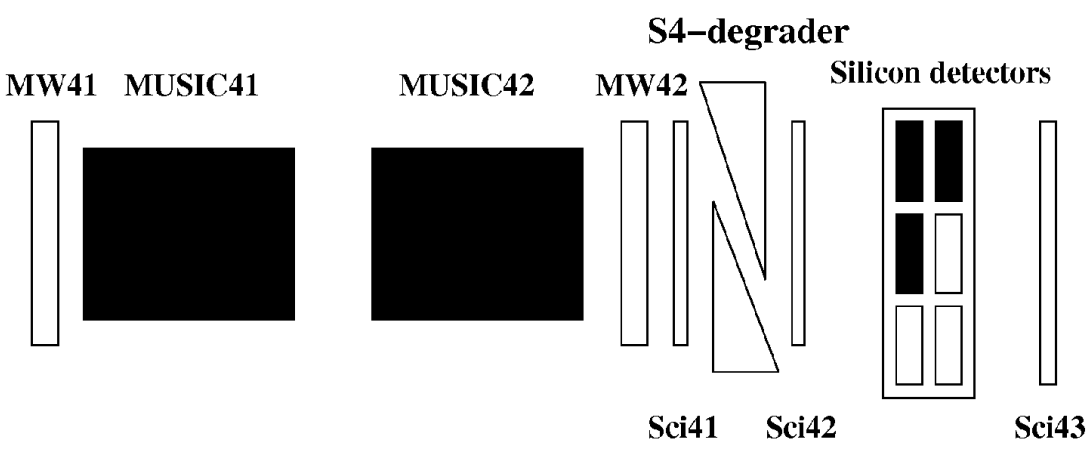

FIGURE 1. Schematic of the detector configuration at the final focus of the GSI Fragment Separator for the first experimental campaign of the Stopped RISING Active Stopper set up. Note that only three of the possible positions (black boxes) in the active stopper were occupied by DSSSDs in the current work. $\mathrm{MW}=$ MultiWire position detectors; $\mathrm{Sci}=$ plastic scintillation detectors. The number '4' corresponds to the detectors being placed at the final focus of the GSI Fragment Separator. The secondary beam products would be moving from left to right on this schematic.

A difficulty with such measurements is that the dynamic range for the secondary reaction products and decay particles are separated by orders-of-magnitude (i.e. $\sim \mathrm{GeVs}$ for the fragments kinetic energies and $\sim 200 \mathrm{keV} \rightarrow$ a few $\mathrm{MeV}$ for the beta-particles). This issue was addressed in the Stopped RISING set up by the use of a semilogarithmic pre-amplifier for the DSSSD energy signals which provided a linear response in the low-energy range (up to $10 \mathrm{MeV}$ ) and a logarithmic response at higher energies $(10 \mathrm{MeV} \rightarrow 3 \mathrm{GeV})$. The linear range of each of the DSSSD strips was calibrated using an open, internal conversion electron ${ }^{207} \mathrm{Bi}$ source while the logarithmic ranges were calibrated for energy response using a pulser. Plastic scintillation detectors were placed in front of and behind the active stopper to give a measure of those fragments which were destroyed in the slowing down process which could then be suppressed in the off-line data analysis. The response of the semilogarithmic pre-amplifier with the RISING Active Stopper is described in more detail in a forthcoming paper [21].

\section{EXPERIMENTAL RESULTS AND DATA ANALYSIS}

The first commissioning experiment for the RISING Active Stopper used a ${ }^{208} \mathrm{~Pb}$ primary beam from the SIS-18 synchrotron at GSI accelerated to an energy of $1 \mathrm{GeV}$ per nucleon. The beam impinged on a $2.5 \mathrm{~g} / \mathrm{cm}^{2}$ thick Be target to produce and study neutron-rich nuclei in the $\mathrm{Hf}, \mathrm{Ta}, \mathrm{W}, \mathrm{Re}$, Os and Ir isotopic chains. The reaction products were transmitted through the FRS and the particle identification was made on 
an event-by-event basis using the standard magnetic rigidity $(B \rho)$, time-of-flight ( $T o F)$, energy loss $(\Delta E)$ and position measurements (see e.g., references $[5,13,22]$ ). A number of different FRS settings were selected to maximise the transmission of a range of neutron-rich nuclei between $\mathrm{A} \sim 170$ and 206.

The study of heavy and exotic neutron-rich species can be hindered in such experiments by the presence of non-fully stripped ions (e.g., hydrogen and helium-like) which are transmitted through the FRS in parallel with fully stripped $(\mathrm{Q}=\mathrm{Z})$ ions. A method to significantly reduce the effect of this so-called A/Q anomaly is outlined in reference [22]. The method uses the deduced energy loss of the ions as they pass through the passive energy degrader at the central focus to determine changes in charge state for ions between the first and second halves of the FRS. This energy loss can be inferred from the change between the measured time-of-flight of the ions in the second half of the FRS and the calculated time-of-flight of the ions in the first half of the separator. Figure 2 shows a 2-D matrix created using data from the current experimental work which was used to select fully stripped ions centred on the transmission of ${ }^{194} \mathrm{Re}$.

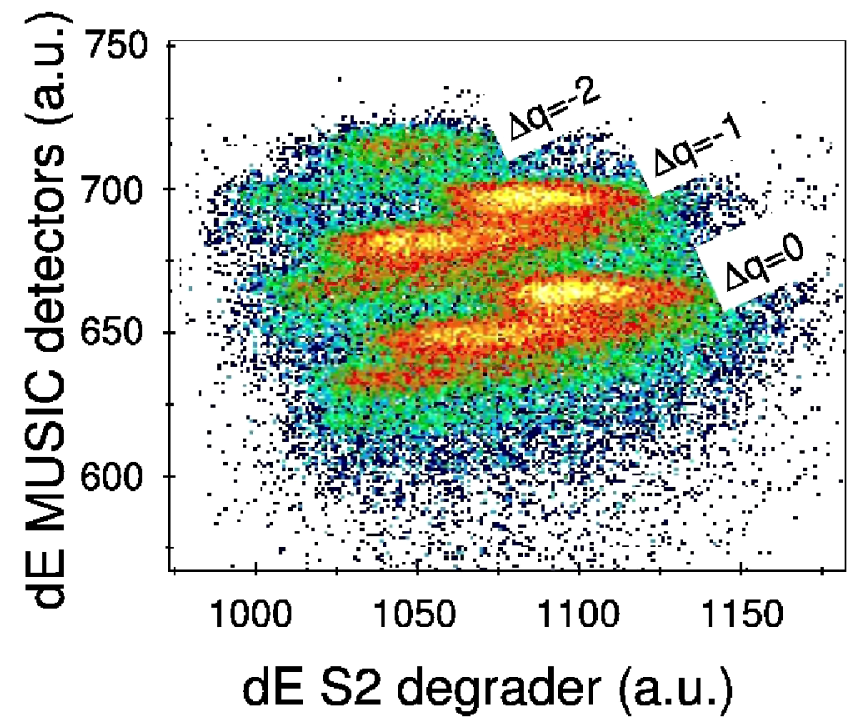

FIGURE 2. Example of a MUSIC energy-loss versus inferred energy loss in the S2 degrader which was used to select fully stripped $(\Delta \mathrm{q}=0){ }^{194} \mathrm{Re}$ ions in the current work.

Gamma-ray decays from secondary beam particles which were transmitted in excited, isomeric states through the FRS can be observed in the RISING gamma-ray array. The characteristic gamma-ray transition energies and decay lifetimes allow the identification of specific nuclear species and can be used as an internal check and calibration of the particle identification procedure. Figure 3 shows examples of gamma-ray and decay curve spectra observed using the RISING gamma-ray array for previously reported $[4,7]$ isomeric states in ${ }^{188} \mathrm{Ta},{ }^{190} \mathrm{~W}$ and ${ }^{192,193} \mathrm{Re}$ which were 
observed in the current work. Note that the decays identified in ${ }^{189} \mathrm{Ta}$ and ${ }^{191} \mathrm{~W}$ in figure 3 have not been previously reported in the literature.

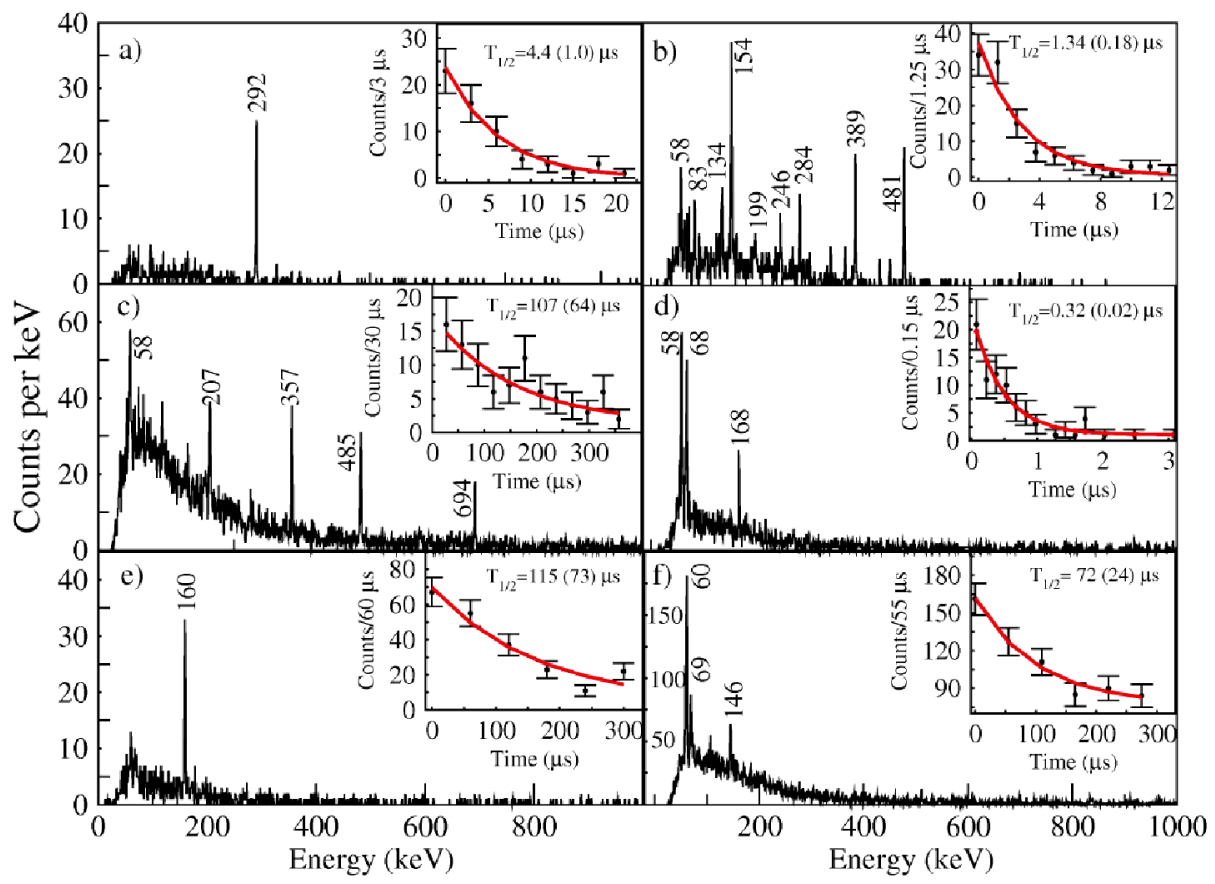

FIGURE 3. Gamma-ray energy and decay time spectra for isomeric decays identified in the current work for decays in (a) ${ }^{188} \mathrm{Ta}$; (b) ${ }^{189} \mathrm{Ta}$; (c) ${ }^{190} \mathrm{~W}$; (d) ${ }^{191} \mathrm{~W}$; (e) ${ }^{192} \mathrm{Re}$; and (f) ${ }^{193} \mathrm{Re}$. The quoted decay lifetimes for these isomers come from least squares fits to the inset decay curves.

\section{BETA-DELAYED SPECTROSCOPY OF FRAGMENTS}

The semi-logarithmic preamplifier allows the responses for both the high-energy primary implant and the lower-energy beta-particle following decay, to be made in the same electronics channel. This allows a pixel by pixel determination of the position of the implanted ion of interest which can be correlated with subsequent decay events in the same and directly neighbouring pixels. By investigating temporally-correlated events with prompt gamma-ray coincidences measured in the Stopped RISING array, beta-delayed gamma-ray spectra can be obtained for specific nuclear species.

Figure 4 shows the example of the gamma-ray spectra in coincidence with beta particles following the radioactive decay of ${ }^{194} \mathrm{Re}$ (i.e., gamma-ray transitions in the daughter ${ }^{194} \mathrm{Os}$ nucleus). The spectrum clearly identifies transitions at energies of 218 and $350 \mathrm{keV}$ which have been previously reported as decays from the yrast states in ${ }^{194}$ Os [23]. The lifetime of the mother nucleus can be determined by the temporal distribution of decays relative to the implants as shown in the inset to figure 4 for ${ }^{194} \mathrm{Re}$. 


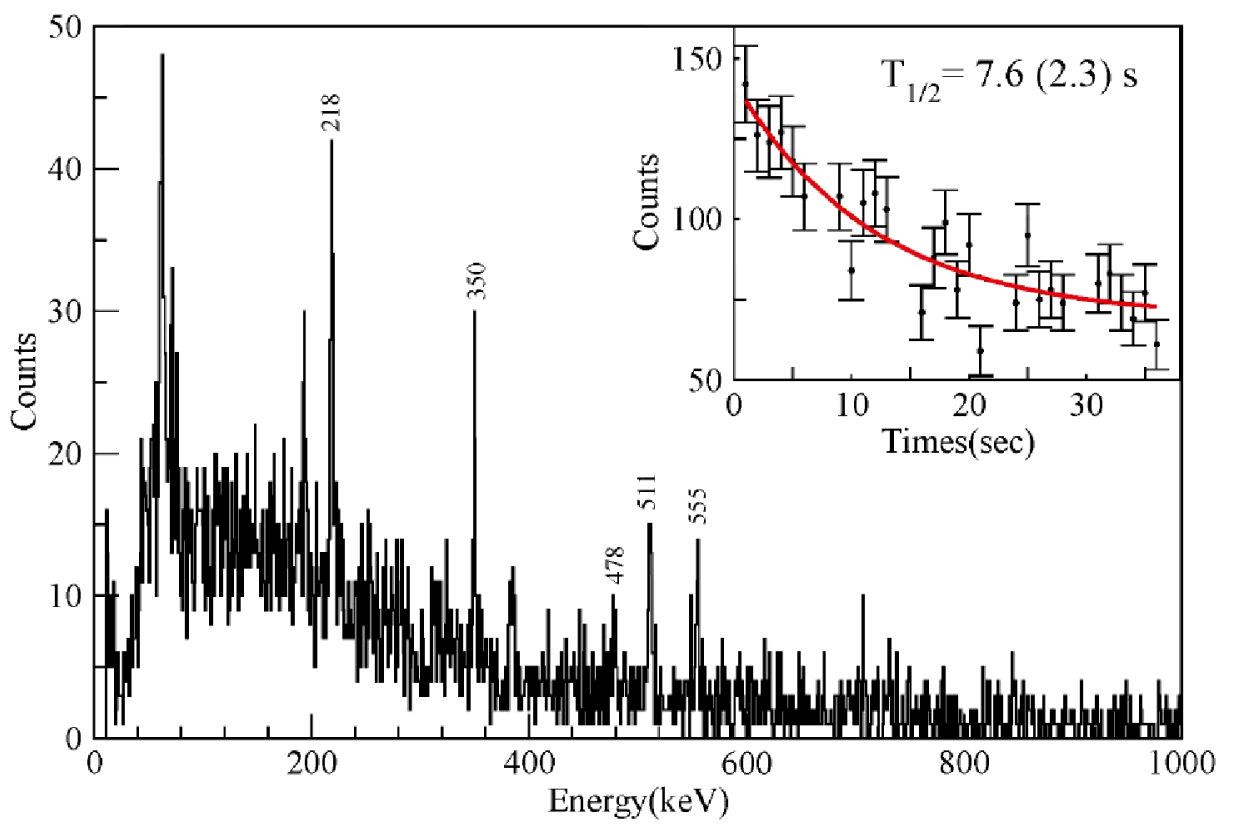

FIGURE 4. Beta-delayed gamma-ray spectrum and lifetime curve for the ${ }^{194} \mathrm{Re} \rightarrow{ }^{194} \mathrm{Os}$ decay.

\section{DISCUSSION OF INITIAL RESULTS}

In cases where the nuclei under study are at the limits of experimental accessibility, excitation energy systematics which can be determined over chains of fixed proton and neutron number can be used to obtain a better global understanding of the evolution of nuclear structure. Figure 5 shows the systematics of the first $2^{+}$excitation energy and the ratio of the excitation energies of the lowest lying $4^{+}$and $2^{+}$states for the even-even $\mathrm{W}$ and Os isotopic chains including data obtained in the current work on ${ }^{194} \mathrm{Os}$ and ${ }^{190} \mathrm{~W}$ [20]. Both the increasing energy of the yrast $2^{+}$energy and the parallel reduction in the energy ratio between the first $4^{+}$and $2^{+}$states points to the reduction in collectivity as the neutron number approaches the $\mathrm{N}=126$ closed shell. The estimate for the quadrupole deformation inferred from the empirically derived relation with the energy of the first $2^{+}$state by Raman et al. [24].

Figure 6 shows the results of new Skyrme Hartree-Fock + BCS shape calculations for the ground states of even-even $\mathrm{W}$ and Os nuclei between $\mathrm{N}=110$ and 118. The calculations were made with no restrictions on the spatial symmetry and show the global minimum for the SkI3 [25] force using the Volume Delta Interaction in the pairing channel [26]. These calculations predict that these nuclei evolve from prolatedeformed nuclei to triaxial systems around neutron number $\mathrm{N}=116$. This is consistent 
with the experimentally deduced ratio of energies of the first $4^{+}$and $2^{+}$states which reduce from a value approaching the idealised axially symmetric rotor limit of 3.33 for the $\mathrm{N}=108$ isotones down to $\sim 2.5$ for the $\mathrm{N}=116$ isotones ${ }^{190} \mathrm{~W}$ and ${ }^{192} \mathrm{Os}$ (see figure 5) indicative of a nucleus with significant triaxial deformation.

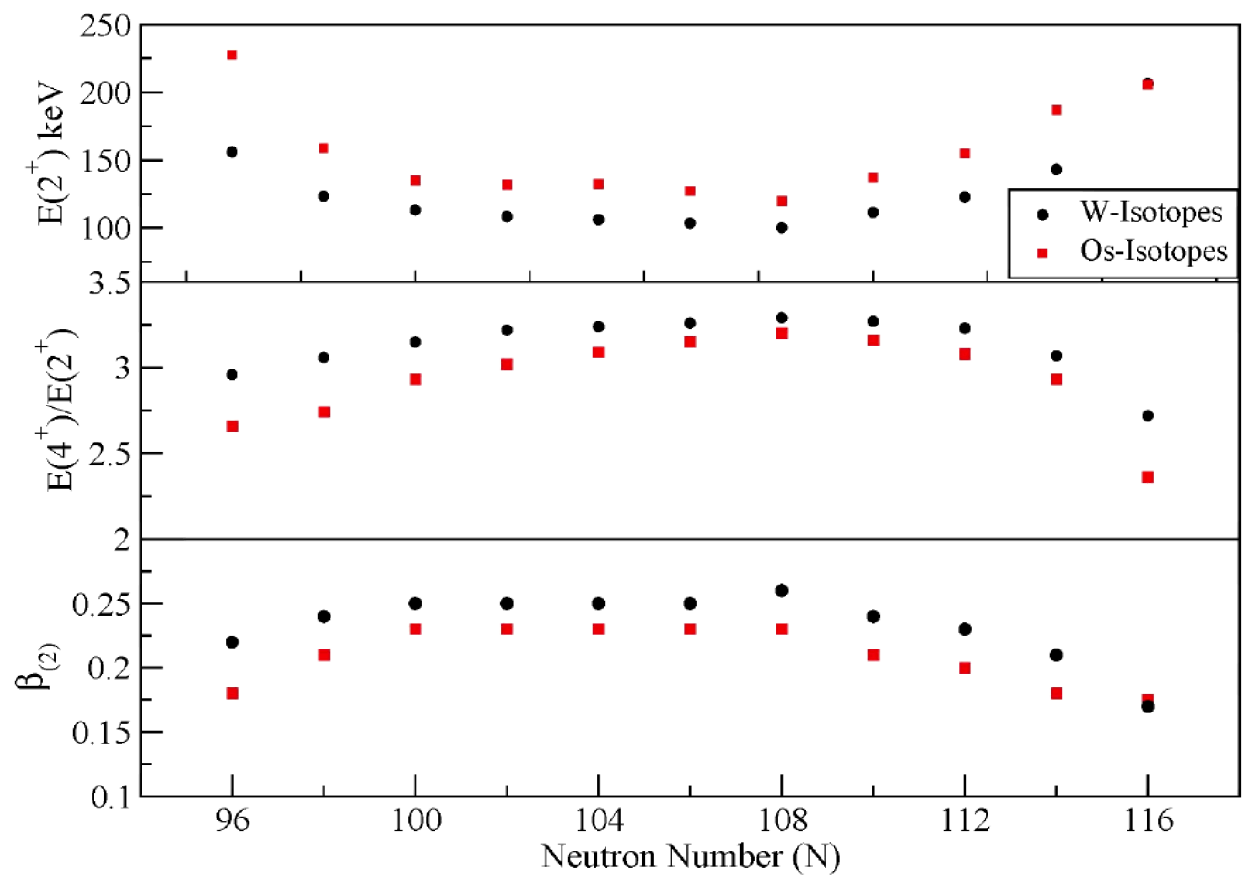

FIGURE 5. Energy systematics of low-lying states in Os and $\mathrm{W}$ isotopes between $\mathrm{N}=96$ and 116.

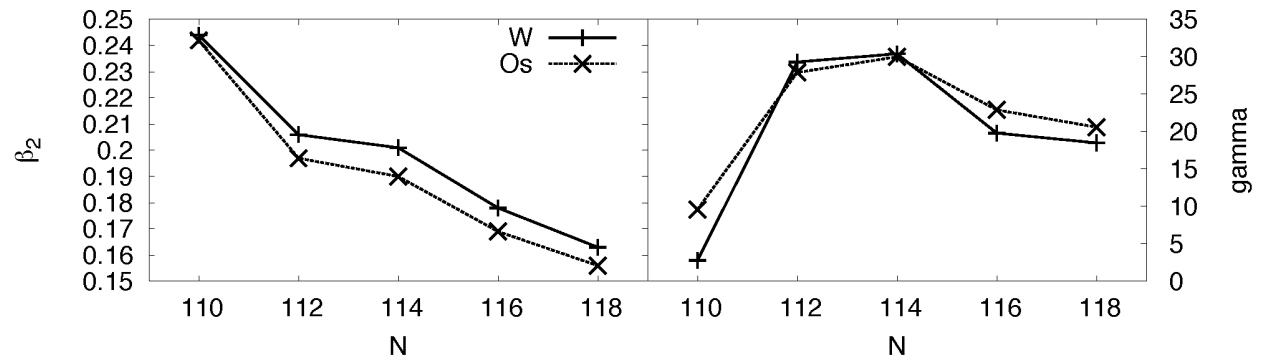

FIGURE 6. Skyrme Hartree-Fock calculations for the ground state quadrupole $\left(\beta_{2}\right)$ and triaxial (gamma) deformations of even-even $\mathrm{W}(\mathrm{Z}=74)$ and $\mathrm{Os}(\mathrm{Z}=76)$ nuclei between $\mathrm{N}=110$ and 118 .

\section{SUMMARY AND FUTURE WORK}

The RISING active stopper has been used for measurements of beta-delayed gamma rays following decays of radioactive nuclei with $\mathrm{A} \sim 190$. The initial data analysis 
shows correlated gamma-ray spectroscopy following the beta-decay of the Rhenium isotopes into their Osmium daughter nuclei and highlights the effectiveness of the RISING Active Stopper in such studies. The spin/parities of states directly populated in the daughter nuclei can then be used with the measured lifetime of the decay to infer the spin/parity of the mother nucleus which can also be used to obtain information on its shape [27]. Other measurements using the active stopper for beta-delayed measurements of fragmentation products of ${ }^{238} \mathrm{U}$ primary beams have also been carried out. This technique expands the regime of nuclei available for spectral study and is of particular significance for heavy neutron-rich nuclei approaching the rapid-neutron process pathway around $\mathrm{N}=126$.

\section{ACKNOWLEDGMENTS}

This work is supported by the EPSRC and STFC (UK), the EU Access to Large Scale Facilities Programme (EURONS, EU contract 506065), The Spanish Ministerio de Educacion y Ciencia and the German BMBF and the AWE plc. (UK).

\section{REFERENCES}

1. A. Gade and T. Glasmacher Prog. Part. Nucl. Phys. 60161 (2008) ; R.F. Casten and B.M. Sherrill Prog. Part. Nucl. Phys. $45 \mathrm{~S} 171$ (2000).

2. H. Geissel, Nucl. Instr. Meth. Phys. Res. B261 1079 (2007).

3. M. Pfützner et al., Phys. Lett. B444 32 (1998).

4. Zs. Podolyák et al., Phys. Lett. B491 225 (2000).

5. M. Pfützner et al., Phys. Rev. C65 064604 (2002).

6. Zs. Podolyák et al., Nucl. Phys. A722 273c (2003).

7. M. Caamano et al., Eur. Phys. J. A23 201 (2005).

8. K.A. Gladnishki et al., Phys. Rev. C69 024617 (2004).

9. Zs. Podolyák et al., Phys. Lett. B632 203 (2006).

10. P.H. Regan et al., Nucl. Phys. A787 491c (2007).

11. A. Jungclaus et al., Phys. Rev. Lett. 99132501 (2007).

12. A.B. Garnsworthy Ph.D. Thesis, University of Surrey, UK (2007).

13. A.B. Garnsworthy et al., Phys. Lett. B660 326 (2008).

14. N.Al-Dahan, Z. Podolyák et al., these proceedings.

15. B. Rubio, W.Gelletly, T. Fujita, A. Algora, A. Gadea et al., private communication.

16. M. Górska et al., Acta. Phys. Pol. B28 1219 (2007).

17. H.J. Wollersheim et al., Nucl. Inst. Meth. Phys. Res. A537 637 (2005).

18. S. Pietri et al., Acta Phys. Pol. B38 1255 (2007).

19. S. Pietri et al., Nucl. Inst. Meth. Phys. Res. B261 1079 (2007).

20. P.H. Regan et al., in press, Int. Jour. Mod. Phys. E, Proceedings of the International Workshop on Nuclear Structure, Shanghai, China (2008).

21. R. Kumar et al., in press, Nucl. Inst. Meth. Phys. Res. A. (2008)

22. J. Benlliure et al., Nucl. Phys. A660 87 (1999); erratum Nucl. Phys. A674 578 (2000).

23. C. Wheldon et al., Phys. Rev. C63 011304 (2001).

24. S. Raman et al., At. Data Nucl. Data Tables 78 1 (2001).

25. P.G. Reinhard and H. Flocard, Nucl. Phys. A584, 467 (1995).

26. M. Bender, P.H. Heenen and P.G. Reinhard Rev. Mod. Phys. 75, 121 (2003).

27. P.M. Walker and F.R. Xu Phys. Rev. C74 067303 (2006). 